\title{
AMILOIDOSIS LOCALIZADA DE VESÍCULAS SEMINALES
}

\author{
L.M. HERRANZ FERNÁNDEZ*, R. ARELLANO GAÑÁN*, SYONGHYUN NAM CHA**, \\ M. JIMÉNEZ GÁLVEZ*, I. PEREIRA SANZ*
}

*Servicio de Urología. **Servicio de Anatomía Patológica. Hospital Universitario de la Princesa. Madrid.

\author{
Actas Urol Esp. 27 (10): 825-828, 2003
}

\section{RESUMEN}

AMILOIDOSIS LOCALIZADA DE VESÍCULAS SEMINALES

La amiloidosis de las vesículas seminales es un hallazgo frecuente en las autopsias, aumentando su incidencia en los individuos de más edad. A pesar de su frecuencia es muy raro que sea sintomática. Presentamos un caso de amiloidosis localizada en las vesículas seminales, sintomático, con hemospermia y dolor suprapúbico, que se diagnosticó mediante biopsias transrectales ecodirigidas. Su presencia obliga a descartar una amiloidosis sistémica. El aumento del tamaño de la vesícula seminal por el depósito de amiloide puede confundirse con una infiltración neoplásica.

PALABRAS CLAVE: Amiloidosis. Vesícula seminal.

\section{ABSTRACT}

SYMPTOMATIC LOCALIZED AMYLOIDOSIS OF THE SEMINAL VESICLES

Amyloidosis of the seminal vesicles is a common finding in autopsies, with increased incidence in older population. It is usually asymptomatic. We report a case of symptomatic localized amyloidosis of the seminal vesicles, with hemospermia and suprapubic pain. Diagnosis was achieved through ultrasound-guided transrectal biopsy. Systemic amyloidosis must be ruled out through proper evaluation. Seminal vesicle enlargement secondary to amyloid deposit may be misdiagnosed as carcinomatous invasion.

KEY WORDS: Amyloidosis. Seminal vesicle.

$\mathrm{E}$ 1 depósito de material amiloide en las vesículas seminales es un hallazgo relativamente frecuente en autopsias con una incidencia entre el 9 al $17 \%$ de los varones estudiados ${ }^{1-7}$. Esta incidencia aumenta con la edad, llegando en los varones mayores de 75 años a una incidencia del 21 al $34 \%^{1-10}$. La mayoría de estos casos son asintomáticos. Presentamos un caso de amiloidosis localizada de las vesículas seminales, en un hombre joven que debutó con hemospermia y dolor suprapúbico.

\section{CASO CLÍNICO}

Paciente de 35 años de edad con antecedentes personales de vasectomía en 1991. Acude en
1997 a nuestra consulta por presentar molestias suprapúbicas y hemospermia persistente. Se le realizan: tacto rectal, análisis de sangre, citologías de semen y orina, uretrocistoscopia con toma de biopsia vesical, ecografía transrectal y resonancia magnética pélvica siendo todas estas pruebas normales. En dos cultivos de semen se obtuvo crecimiento bacteriano de E. Faecalis $\left(1^{\circ}\right)$ y S. Agalactiae $\left(2^{\circ}\right)$. Fue tratado con antibióticos orales y desaparecieron los síntomas.

En el 2002 vuelve a visitar nuestra consulta por comenzar nuevamente con hemospermia, que se repite en todas las ocasiones en que eyacula. En el tacto rectal presenta una próstata de un volumen I/IV, fibroelástica, simétrica y uniforme. 
El análisis de sangre es normal (PSA: 0,40 $\mathrm{ng} / \mathrm{ml})$. Los cultivos de orina, semen y secreción prostática son negativos. En la citología del semen se observa un fondo hemático sin espermatozoides y sin componente inflamatorio. La ecografía abdominal es normal. En la ecografía transrectal se visualiza una vesícula seminal izquierda (VSI) dilatada con septos en su interior; vesícula seminal derecha (VSD) y próstata normales (Fig. 1). Se decide realizar una PAAF transrectal de dicha VSI no obteniéndose material en la aspiración. Se realizan biopsias transrectales ecodirigidas de dichas vesículas en cuyo análisis histológico se observa depósito de amiloide en ambas vesículas que se tiñe con rojo Congo y con luz polarizada presentaba birrefringencia verdemanzana (Figs. 2 y 3). Se obtuvo también tejido prostático normal. Debido a la escasez de tejido no se pudo completar el estudio para la tipificación del amiloide.

El enfermo se remite a la consulta de Medicina Interna de nuestro Hospital, donde es evaluado descartándose una amiloidosis sistémica. El paciente continúa con hemospermia y se le ofrece tratamiento quirúrgico que rechaza en principio, prefiriendo seguir revisiones periódicas.

\section{DISCUSIÓN}

La amiloidosis es una entidad de etiología desconocida que se caracteriza por depósitos extracelulares de material proteináceo, hialino, homo-

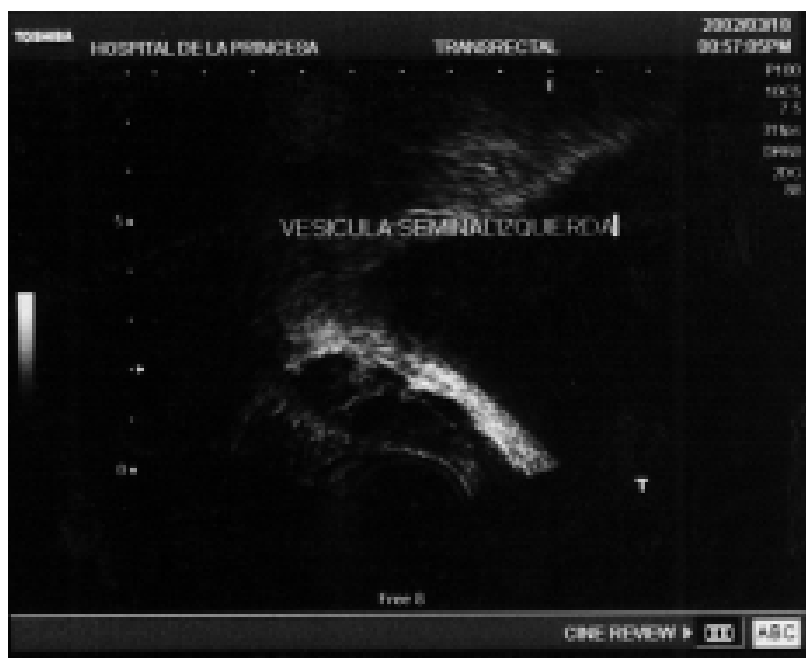

FIGURA 1. Ecografia transrectal: vesicula seminal izquierda dilatada con septos en su interior.

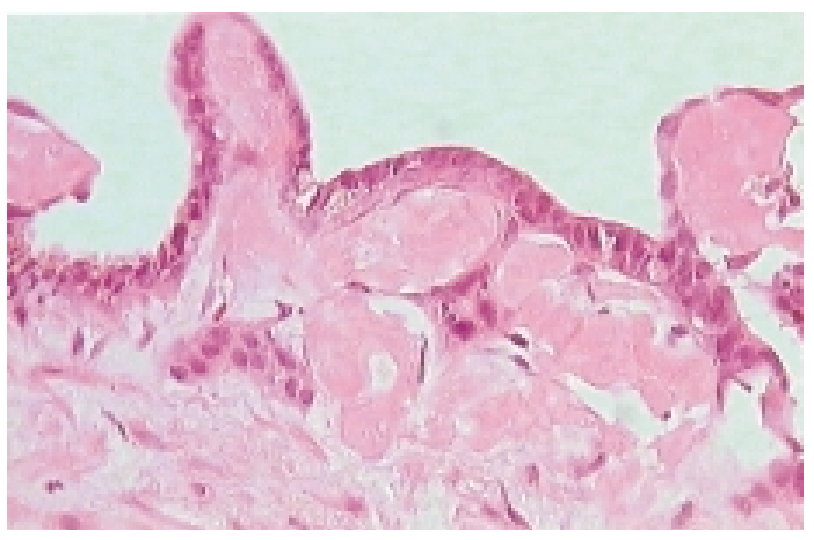

FIGURA 2. Vesicula seminal. Tinción de hematoxilina/ eosina. Depósito subepitelial de un material acelular, homogéneo y eosinófilo.

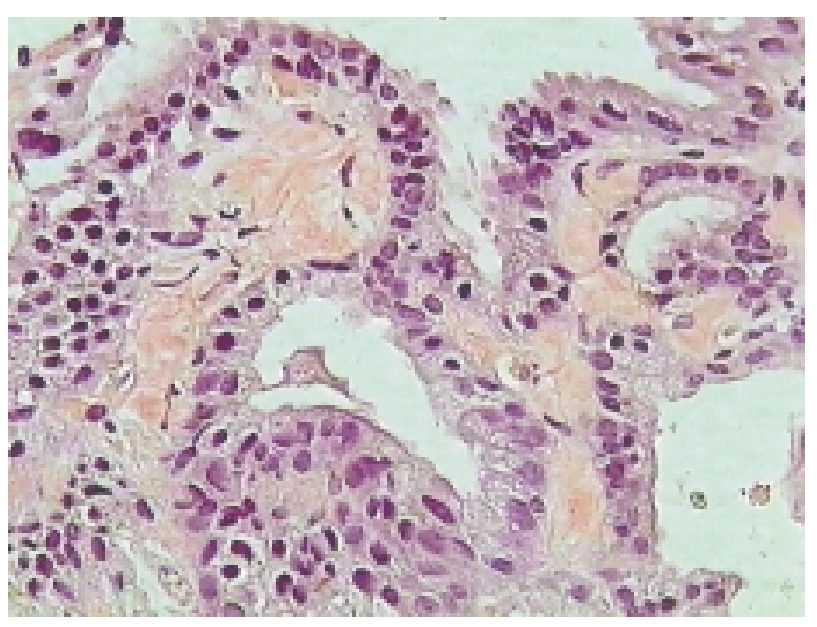

FIGURA 3. Vesícula seminal. Tinción con rojo Congo. Material acelular, subepitelial que se tiñe con dicho colorante.

géneo y eosinofilico. Dicha sustancia se tiñe con el rojo Congo, muestra una birrefringencia verdemanzana con luz polarizada y tiene una ultraestructura fibrilar característica al microscopio electrónico ${ }^{1,5,7,11,12}$.

Esta entidad la podemos dividir en amiloidosis sistémica que incluye las discrasias de inmunocitos con amiloidosis (amiloidosis primaria), amiloidosis sistémica reactiva (amiloidosis secundaria), amiloidosis asociada a hemodiálisis y amiloidosis hereditaria; y amiloidosis localizada que puede afectar a cualquier órgano. La amiloidosis senil puede cursar tanto de forma sistémica como localizada ${ }^{5,7,9,11}$.

La afectación de las vesículas seminales por la amiloidosis es un hallazgo de relativa frecuencia 
en autopsias, variando la incidencia en los distintos estudios realizados entre el 9 y el $17 \%^{1-7}$. $\mathrm{Su}$ presencia aumenta con la edad, llegando a tener una incidencia del 21 al 34\% en los varones mayores de 75 años $^{1-10}$. Esta amiloidosis localizada en las vesículas seminales es por tanto, edad dependiente ${ }^{1-5,7-10,12,13}$. La presencia de depósito de amiloide en las vesículas seminales puede limitarse a éstas o asociarse a amiloidosis sistémica. Con frecuencia la forma localizada de amiloidosis en vesícula seminal se asocia a pacientes de avanzada edad, por lo que también se considera una manifestación de la amiloidosis senil. En la amiloidosis localizada observamos un acúmulo subepitelial de amiloide tipo AA. En la forma sistémica podemos encontrar depósito amiloide en vasos y tejido muscular de tipo AA o $\mathrm{AL}$, en función de la etiopatogenia de la amiloidosis $^{1-9,12-13}$.

La amiloidosis localizada de las vesículas seminales suele ser asintomática, existiendo muy pocos casos que hayan manifestado algún tipo de sintomatología, como en nuestro caso. Se han comunicado hemospermia, dolor perineal o suprapúbico crónico y síntomas obstructi$\operatorname{vos}^{7,8,14}$

Nuestro caso presentaba hemospermia. La hemospermia se produce por amiloidosis de las vesículas seminales en un 0 a un $2,3 \%$ de los casos, según las series ${ }^{14,15}$. Para explicar este sangrado se han expuesto varias teorias. Unos autores defienden que la vesícula con depósito de amiloide sangra por un mecanismo parecido al que ocurre en la hemorragia vesical "ex-vacuo", al vaciarse la vesícula seminal rápidamente con el eyaculado. Otros proponen la posibilidad de la creación de una fístula entre la vena iliaca y la vesícula seminal ${ }^{14}$. Por último, otros autores defienden la posibilidad de alteración de los vasos sanguíneos al lesionarse la pared por el amiloide ${ }^{9}$.

Se han publicado varios casos de tumores de próstata o vejiga en los que al hacer el estudio de extensión mediante una RMN se han encontrado unas vesículas seminales dilatadas con las paredes gruesas, siendo etiquetados de neoplasias localmente avanzadas, no ofreciendo tratamientos curativos, cuando lo que verdaderamente presentaban estos casos era una amiloidosis de las vesículas seminales. Por ello, pensamos que debe tenerse en cuenta dicha posibilidad en el diagnóstico de extensión de estos tumores para evitar la sobre-estadificación ${ }^{1,3,8,16,17}$.

\section{CONCLUSIONES}

La amiloidosis de las vesículas seminales tiene una incidencia relativamente frecuente en los estudios realizados con autopsias de varones, siendo la mayoría de los casos asintomáticos. Ante el diagnóstico de una amiloidosis de vesícula seminal se debe diferenciar si nos encontramos ante una amiloidosis sistémica o localizada. También es importante en el diagnóstico diferencial de hemospermia tener en cuenta esta patología como posible causante del sangrado. Por último, en estudios de extensión de una neoplasia vesical o prostática, tener en cuenta que el aumento de tamaño de las vesículas seminales puede ser producido por el depósito de material amiloide.

\section{REFERENCIAS}

1. JAGER GJ, RUIJTER ETG, DE LA ROSETTE JJMCH, VAN DE KAA CHA.: Amyloidosis of the seminal vesicles simulating tumor invasion of prostatic carcinoma on endorectal MR images. Eur Radiol 1997; 7: 552-554.

2. COINÉ JD, KEALY WF.: Seminal vesicle amyloidosis: morphological, histochemical and immunohistochemical observations. Histopathology 1993; 22: 173176.

3. RAMCHANDANI P, SCHNALL MD, LIVOLSI VA, TOMASZEWSKI JE, POLLACK HM.: Senile amyloidosis of the seminal vesicles mimicking metastatic spread of prostatic carcinoma on MR images. AJR 1993; 161: 99-100.

4. GOLDMAN H.: Amyloidosis of seminal vesicles and vas deferens. Arch Path 1963; 75: 94-98.

5. PITKANEN P, WESTERMARK P, CORNWELL GG $3^{\text {rd }}$, MURDOCH W.: Amyloid of the seminal vesicles. A distinctive and common localized form of senile amyloidosis. Am J Pathol 1983; 110 (1): 64-69.

6. SUESS K, MOCH H, EPPER R, KOLLER A, DURMULLER U, MIHATSCH MJ.: Heterogeneity of seminal vesicle amyloid. Immunohistochemical detection of lactoferrin and amyloid of the prealbumin-transthyretin type. Pathologe 1998; 19 (2): 115-119.

7. CARRIS CK, MCLAUGHLIN AP, GITTES RF.: Amyloidosis of the lower genitourinary tract. $J$ Urol 1976; 115: 423-426.

8. BOTASH RJ, POSTER RB, ABRAHAM JL, MAKHULI ZM.: Senile seminal vesicle amyloidosis associated with hematospermia: demonstration by endorectal MRI. J Comput Assist Tomogr 1997; 21 (5): 748-749.

9. MCDONALD JH, HECKEL NJ.: Primary amyloidosis of the lower genitourinary tract. $J$ Urol 1956; 75: 122-132. 
10. SEROV VV.: Local forms of amyloidosis as a manifestation of age-related pathology: a new look at the problem. Arkh Patol 1994; 56 (5): 39-43.

11. TRÍVEZ BONED MA, BLAS MARÍN M, GARCÍA GARCÍA MA, GIL MARTÍNEZ P, GARCÍA DE JALÓN MARTÍNEZ A, RIOJA SANZ LA.: Amiloidosis localizada de la uretra. Actas Urol Esp 2002; 26 (1): 4649.

12. SEIDMAN JD, SHMOOKLER BM, CONNOLLY B, LACK EE.: Localized amiloidosis of seminal vesicles: report of three cases in surgically obtained material. Mod Pathol 1989; 2 (6): 671-675.

13. ESSLIMANI M, SERRE I, GRANIER M, ROBERT M, BALDET P, COSTES V.: Urogenital amyloidosis: clinico-pathological study of 8 cases. Ann Pathol 1999; 19 (6): 487-491.

14. FLETCHER MS, HERZBERG $Z$, PRYOR JP.: The aetiology and investigation of haemospermia. $\mathrm{Br} J$ Urol 1981; 53 (6): 669-671.
15. YU HM, WONG KK, LIM TK, LEONG CH.: Clinical study of hemospermia. Urology 1977; 10 (6): 562563.

16. HUTTER BE, HUCH BONI RA, HAWELSKI S, MAURER R, KRESTIN GP.: Amyloidosis simulating tumor infiltration of the seminal vesicles. Rofo Fortschr Geb Rontgenstr Neuen Bildgeb Verfahr 1995; 163 (2): 184-185.

17. KAJI Y, SUGIMURA K, NAGAOKA S, ISHIDA T.: Amyloid deposition in seminal vesicles mimicking tumor invasion from bladder cancer: MR findings. J Comput Assist Tomogr 1992; 16 (6): 989-991.

Dr. L.M. Herranz Fernández Servicio de Urología. Hospital de la Princesa C/ Diego de León, 62 - 28006 Madrid

(Trabajo recibido el 29 enero de 2003) 